\title{
A Rare Case of Septic Arthritis of Lumbar Facet Joint with Epidural Abscess, and Bacterial Meningitis Caused by Methicillin-Sensitive Staphylococcus aureus
}

\author{
Miguel Maldonado-Morán ${ }^{1}$ \\ Juan M. Muñoz ${ }^{2}$ \\ Jairo M. Mejía ${ }^{6}$
${ }^{1}$ Department of Neuroscience, Universidad Militar Nueva Granada, Bogotá, Colombia Bogotá, Colombia
${ }^{3}$ Department of Orthopaedics, Spine Services, Universidad Militar Nueva Granada, Facultad de Medicina y Ciencias de la Salud, Bogotá, Colombia
${ }^{4}$ Department of Orthopaedics, Spine Services, Universidad Militar Nueva Granada, Facultad de Medicina y Ciencias de la Salud, Bogotá, Colombia
${ }^{5}$ Movement Disorder Specialist Neurology, University of Florida, Gainesville, Florida, United States
${ }^{6}$ Department of Neurology, Universidad Militar Nueva Granada, \\ 2Department of Neurosurgery, Universidad Militar Nueva Granada, \\ Bogotá, Colombia
}

Javier I. Matta

Victor M. Arrieta ${ }^{4}$

Oscar P. Bernal ${ }^{5}$

\author{
Address for correspondence Miguel Maldonado-Morán, MD \\ Universidad Militar Nueva Granada, Bogotá, Colombia \\ (e-mail: miguel.mamoran@gmail.com).
}

Indian J Neurosurg 2018;7:139-141

\begin{abstract}
Keywords

- septic arthritis

- facet joint

- epidural abscess

- meningitis

- Staphylococcus aureus

Septic arthritis of the facet joint is an extremely rare condition, even more in young immunocompetent patients. There have been approximately only 40 cases of this entity reported worldwide. Here, the authors present a 16-year-old male patient with lower back pain, fever, and cephalea. Blood cultures and cerebrospinal fluid analysis confirmed methicillin-sensitive Staphylococcus aureus infection, and magnetic resonance imaging showed septic arthritis of the lumbar left facet joint L3 and epidural abscess. Intravenous oxacillin was administered, and the patient improved. No other treatment was required.
\end{abstract}

\section{Introduction}

First described in 1966, septic arthritis is an extremely rare entity. ${ }^{1}$ Nowadays, only 40 cases of pyogenic arthritis of the facet joint are reported worldwide. ${ }^{2}$ Most of the pyogenic infections of the spine comes from hematogenous dissemination, and the direct dissemination to the paraspinal soft tissue occurs in the $20 \%$ of the cases. ${ }^{1}$

\section{Case Report}

We present a case of a 16-year-old male patient, who presented to the hospital with lower back pain of 1 month of apparition. The patient informed us that he could not make normal movements because of severe pain and that in the last days it was associated with headache and fever. His medical history shows he is being treated with folic acid, as he suffers from hereditary spherocytosis. There was no history of urinary incontinence, spinal trauma, or any other medical problem.

On clinical examination, we found a febrile and tachycardic patient, with nuchal rigidity, muscular strength lower limbs was 5/5, sensation and reflexes were normal, and Lasègue's test was negative.

In the first paraclinical test, it was evidenced leukocytosis (23.720), with neutrophilia (84\%), an elevated C-reactive protein (18), and a normal urinalysis. As the patient's condition was getting worse, and by the clinical suspicion, we decided to take a blood culture and do a lumbar puncture. In the blood culture, we found methicillin-sensitive Staphylococcus aureus, and in the analysis of lumbar puncture, 2,160 leukocytes, $100 \%$ neutrophils, $153 \mathrm{mg} / \mathrm{dL}$ proteins, and $53 \mathrm{mg} / \mathrm{dL}$
License terms

Society of India 
of glucose, with methicillin-sensitive $S$. aureus growing in the culture of cerebrospinal fluid.

Magnetic resonance imaging (MRI) study of the lumbosacral spine revealed in left L3 osteoarticular inflammatory changes associated with epidural abscess ( - Figs. 1 and 2 ).

The patient was treated with conservative management with intravenous oxacillin, because he did not meet criteria for surgery. His evolution was satisfactory with no other additional treatment.

\section{Discussion}

Septic arthritis of the lumbar facet joint is an infrequent condition. It has been reported in older patients and those who are immune-compromised, but in the literature, cases of young immune-competent patients can also be found. Although the precise pathogenesis of the septic arthritis of the lumbar facet joints is not clear, some risk factors such as diabetes mellitus and extraspinal infections have been proposed, and hematogenous spread is the leading cause. ${ }^{3}$ In other reports, the authors show that it can be secondary to vertebral body osteomyelitis, epidural abscess, paraspinal abscess, and even be a complication of a steroid injection., ${ }^{2,4}$

According to reported epidemiology, the most common organisms causing septic arthritis of the facet joints by hematogenous spread are $S$. aureus (70\% of the cases) and Staphylococcus epidermidis., ${ }^{2,5}$ Other bacterial organisms such as Streptococcus and gram-negative rods are also reported. ${ }^{7}$ Inflammatory markers including C-reactive protein and erythrocyte sedimentation rate are consistently raised, with white blood count raised in $50 \%$ of the cases and, in the same percentage, positive blood cultures. ${ }^{2,3,6,8}$

The physiopathology of the abscess can be explained because the facet joint cavity is narrow, and the infection can easily spread to the epidural space by rupture of the ventral aspect of the joint capsule and the paravertebral muscles by rupture of the posterior aspect of the joint capsule. ${ }^{5}$

Diagnosis of this entity is difficult because it can behave as a degenerative disc disease and spondylosis. ${ }^{9} \mathrm{MRI}$ is the elective imaging modality of choice; it is both sensitive and specific within 2 days of infection, and when it is contrasted with technetium 99 , it has $100 \%$ sensitivity for facet joint compromise. ${ }^{9,10}$ The radiologic features in MRI of the spine are joint effusion, periarticular bone marrow edema, and periarticular soft tissue edema with enhancement. ${ }^{9}$ Diagnostic confirmation is said to be performed through bone biopsy or culture of the bacteria. However, many times, if the facet compromise is isolated, the possibility of a negative biopsy is high. ${ }^{2}$ According to the articles reviewed, it is recommended that if blood cultures are negative or if there is doubt in the diagnosis of septic arthritis of the facet joint, a culture must be performed through needle aspiration at the level of the facet under fluoroscope or ultrasound. ${ }^{7}$

Differentiating a vertebral osteomyelitis from a septic facet joint arthritis is difficult by clinical and laboratory tests, but MRI of the spine has facilitated this and its early detection. It is recalled that changes in the intensities of the MRI suggestive for edema and epidural abscess can be detected in the first week. The use of scintigraphy in this pathology is limited, because it is very sensitive but not very specific for the diagnosis of pyogenic facet joint arthritis. ${ }^{5}$
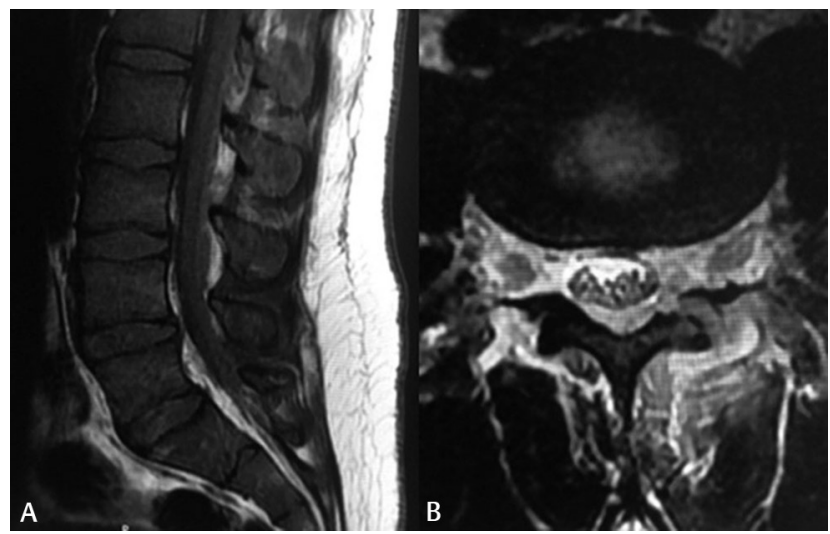

Fig. 2 (A) Sagittal view of magnetic resonance imaging showing an epidural abscess. (B) T2-weighted image, axial view. Nerve roots clumped together, which suggests epidural abscess.

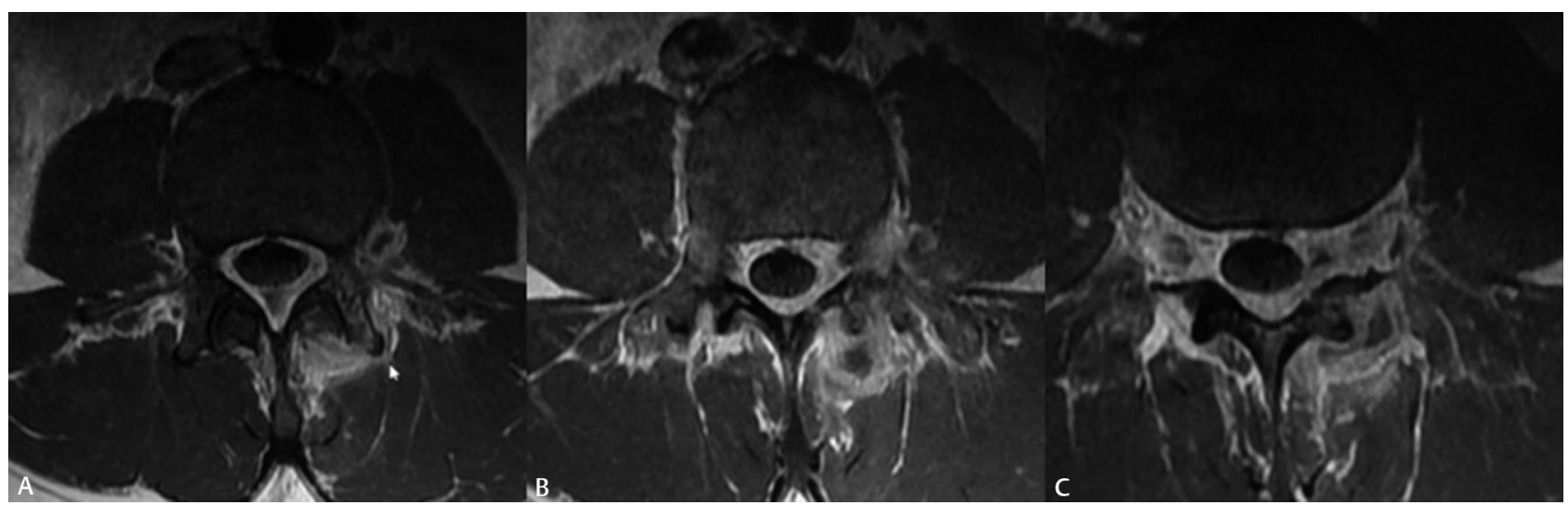

Fig. 1 (A-C) Axial view lumbar magnetic resonance imaging. Changes in the left L3 facet joint intensities that suggest an inflammatory process, with changes in the surrounding tissues. 
The clinical indications reported in the literature of pyogenic arthritis of the facet joint includes pain in the region of the persistent spine that does not improve with rest and fever. Nearly $90 \%$ of cases report localized pain in the spine and $50 \%$ are associated with fever. ${ }^{2}$

The treatment of choice is conservative management with intravenous antibiotics. It is recommended for 2 weeks intravenously and then 4 weeks orally. In addition, it should be followed up with acute phase reactants and imaging. ${ }^{9}$ Surgical or open arthroscopic drainage with debridement is only reserved for patients with refractoriness to previous antibiotic management or acute neurologic compromise. ${ }^{11}$ Regarding the dissemination of bacterial pyogenic arthritis to the paravertebral muscles and epidural space, it often does not require surgical management. Only the patient's antibiotic management can improve it. 5,12

\section{Conflict of Interest}

None.

\section{References}

1 Swayne LC, Dorsky S, Caruana V, Kaplan IL. Septic arthritis of a lumbar facet joint: detection with bone SPECT imaging. J Nucl Med 1989;30(8):1408-1411

2 Rhyu KW, Park SE, Ji JH, Park I, Kim YY. Pyogenic arthritis of the facet joint with concurrent epidural and paraspinal abscess: a case report. Asian Spine J 2011;5(4):245-249
3 Muffoletto AJ, Ketonen LM, Mader JT, Crow WN, Hadjipavlou AG. Hematogenous pyogenic facet joint infection. Spine 2001;26(14):1570-1576

4 Lee JC, Doh HW, Cho YI, Kim KJ, Kim YI, Shin BJ. Pyogenic arthritis and paraspinal abscess following facet joint steroid injection: a case report. J Korean Soc Spine Surg 2003;10:196-201

5 Ogura T, Mikami Y, Hase H, Mori M, Hayashida T, Kubo T. Septic arthritis of a lumbar facet joint associated with epidural and paraspinal abscess. Orthopedics 2005;28(2):173-175

6 Rombauts PA, Linden PM, Buyse AJ, Snoecx MP, Lysens RJ, Gryspeerdt SS. Septic arthritis of a lumbar facet joint caused by Staphylococcus aureus. Spine 2000;25(13):1736-1738

7 Ergan M, Macro M, Benhamou CL, et al. Septic arthritis of lumbar facet joints. A review of six cases. Rev Rhum Engl Ed 1997;64(6):386-395

8 Calderone RR, Larsen JM. Overview and classification of spinal infections. Orthop Clin North Am 1996;27(1):1-8

9 Rajeev A, Choudhry N, Shaikh M, Newby M. Lumbar facet joint septic arthritis presenting atypically as acute abdomen-a case report and review of the literature. Int J Surg Case Rep 2016;25:243-245

10 Pilleul F, Garcia J. Septic arthritis of the spine facet joint: early positive diagnosis on magnetic resonance imaging. Review of two cases. Joint Bone Spine 2000;67(3):234-237

11 Stecher JM, El-Khoury GY, Hitchon PW. Cervical facet joint septic arthritis: a case report. Iowa Orthop J 2010;30:182-187

12 Magee M, Kannangara S, Dennien B, Lonergan R, Emmett L, Van der Wall H. Paraspinal abscess complicating facet joint injection. Clin Nucl Med 2000;25(1):71-73 